BULLETIN Bulletin hispanique

HISPANIQUE Université Michel de Montaigne Bordeaux

115-1 | 2013

Poésie et société en Espagne : 1650-1750

\title{
Sobre la redacción de La Eneida de Virgilio traducida por Graciliano Afonso
}

\section{Francisco Salas Salgado}

\section{(2) OpenEdition}

Journals

\section{Edición electrónica}

URL: http://journals.openedition.org/bulletinhispanique/2503

DOI: 10.4000/bulletinhispanique.2503

ISSN: 1775-3821

\section{Editor}

Presses universitaires de Bordeaux

\section{Edición impresa}

Fecha de publicación: 1 junio 2013

Paginación: 285-304

ISBN: 978-2-86781-898-1

ISSN: 0007-4640

\section{Referencia electrónica}

Francisco Salas Salgado, «Sobre la redacción de La Eneida de Virgilio traducida por Graciliano Afonso », Bulletin hispanique [En línea], 115-1 | 2013, Publicado el 01 junio 2016, consultado el 07 mayo 2019.

URL : http://journals.openedition.org/bulletinhispanique/2503; DOI : 10.4000/bulletinhispanique.2503 


\title{
Sobre la redacción de La Eneida de Virgilio traducida por Graciliano Afonso
}

\author{
Francisco Salas Salgado \\ Universidad de La Laguna
}

En 1854 paraît, publiée à Las Palmas de Gran Canaria (Canaries), une traduction de l'Enéide de Virgile. Son auteur, Graciliano Afonso Naranjo (1775-1861) a laissé une ample production littéraire, mais aussi de nombreuses traductions de classiques grecs et latins. La découverte des manuscrits antérieurs au texte imprimé, permet d'étudier le processus de rédaction d'une cuvre selon toute évidence amplement lue dans le périmètre insulaire.

Mots-Clés: traduction des classiques, tradition textuelle, $\mathrm{XIX}^{\mathrm{e}}$ siècle.

En 1854 apareció publicada en Las Palmas de Gran Canaria (Islas Canarias) la traducción de la Eneida de Virgilio. Su autor, Graciliano Afonso Naranjo (1775-1861) dejó una amplia producción literaria, pero también abundantes traducciones de clásicos grecolatinos. El descubrimiento de manuscritos previos al texto impreso de esta traducción posibilita estudiar el proceso de redacción de una obra que con toda seguridad fue ampliamente leída en el ámbito insular.

Palabras claves: traducción de autores clásicos, tradición textual, siglo XIX.

In 1854, the translation of the Eneida by Virgil was published in Las Palmas de Gran Canarias (Canary Islands). Its author, Graciliano Afonso Naranjo (1775-1861), left a wide literary production, but also many translations of classic authors Latin as Greek. The discovery of manuscripts anterior to the printed text of this translation makes it possible to study the process of draft in a work that was certainly widely read in the insular area.

Keywords: translation of classical authors, textual tradition, Nineteenth Century.

\section{INTRODUCCIÓN}

La Eneida, el gran poema épico de Virgilio, y una de las más importantes obras que nos ha legado la Antigüedad, fue objeto desde temprano de ediciones, comentarios y traducciones. Son precisamente estas últimas, las traducciones, 
realizadas de las lenguas clásicas a las diferentes lenguas nacionales, las que contribuyeron a que las andanzas de Eneas y los troyanos fueran leídas por todos aquellos cuyo conocimiento de la lengua latina era insuficiente. Se posibilitaba de esta manera la recuperación del mundo clásico, y por ende la asunción de unos determinados valores, por parte de la mayoría de las sociedades cultas, cuyos escritores, siguiendo a G. Highet, «se volvían a Roma y a Grecia en busca de relatos interesantes y no tan conocidos, en busca de ideas nuevas y eficaces» ${ }^{1}$.

Traductores y traducciones de la Eneida ha habido muchos en todos lados y en todos los momentos. Algunas de estas traducciones fueron tan celebradas que tuvieron una vigencia más allá de la época en que fueron compuestas. En el caso de España, ocurre esto, por poner un ejemplo, con la traducción de Gregorio Hernández de Velasco, que seguramente leyeron los clásicos españoles, desde Lope de Vega hasta Cervantes, y que según los datos que ofrece J. de EchaveSustaeta recibió catorce ediciones hasta el año $1838^{2}$. Sin embargo, se entiende que existan muchas otras versiones que propiciaron el conocimiento de este poema virgiliano en lugares donde también se dio (o, por lo menos, se intentó) esa recuperación del mundo clásico; lugares alejados, incluso, geográficamente, y donde por motivos diversos dicha recuperación no se dio de inmediato.

Esta última apreciación tiene mucho que ver con el tema que me propongo tratar aquí, relacionado con la expansión de la literatura clásica en las Islas Canarias, de la que existen, como se sabe y se ha estudiado, referencias en los propios autores grecolatinos ${ }^{3}$. En cambio es mucho lo que queda por hacer sobre la pervivencia de las letras latinas y griegas entre los escritores nacidos en esas tierras ${ }^{4}$.

Las Islas Canarias fueron conquistadas e incorporadas a la Corona de Castilla a finales del siglo $\mathrm{XV}^{5}$. Es lógico pensar que entonces no se diera un ambiente que propiciara la enseñanza y el cultivo de las humanidades clásicas, principalmente de la lengua latina, fundamental en la época como medio de

1. Gilbert Highet, La tradición clásica. Influencias griegas y romanas en la literatura occidental, trad. española de Antonio Alatorre, t. I, México, Fondo de Cultura Económica, 1986, p. 7.

2. Cf. Javier de Echave-Sustaeta, Virgilio y nosotros, Barcelona, Cefiso, 1964, p. 108. Para las traducciones de Virgilio en España es de obligada consulta Marcelino Menéndez Pelayo, Bibliografía hispano-latina clásica, ed. preparada por Enrique Sánchez Reyes, t. VIII, Santander, C.S.I.C, Aldus, 1952, pp. 194-397. Recientemente puede verse Vicente Cristóbal López-José Luis Vidal, «Virgilio», en Francisco Lafarga - Luis Pegenaute (eds.), Diccionario histórico de la traducción en España, Madrid, Gredos, 2009, pp. 1156-116.

3. Cf. de manera general, Antonio Cabrera Perera, Las Islas Canarias en el Mundo Clásico, Islas Canarias, Viceconsejería de Cultura y Deportes del Gobierno de Canarias, 1988; Marcos Martínez Hernández, Canarias en la Mitología, Santa Cruz de Tenerife, Centro de la Cultura Popular Canaria, 1992; y, del mismo autor, Las Islas Canarias de la Antigüedad al Renacimiento, Santa Cruz de Tenerife, Centro de la Cultura Popular Canaria, 1996.

4. Sobre el tema véase Francisco Salas Salgado, Humanistas canarios de los siglos XVI a XIX. T. I. Contexto histórico-literario. T. II. Catálogo biobibliográfico, Universidad de La Laguna, Servicio de Publicaciones, 1999.

5. Cf. sobre este asunto Eduardo Aznar Vallejo, La integración de las Islas Canarias en la Corona de Castilla (1478-1526), Las Palmas de Gran Canaria, Ediciones del Cabildo Insular de Gran Canaria, 1992. 
comunicación. La principal atención derivaría hacia otros aspectos menos trascendentales y más mundanos. No obstante, el aspecto educativo no se descuidó, y para el conocimiento de la lengua latina se crearon determinadas Cátedras de Gramática, algunas al amparo de la Iglesia y otras de signo laico ${ }^{6}$. Las producciones humanísticas que se realizaron entonces (entendiendo aquí no sólo los escritos neolatinos), verdadero barómetro de la extensión de esa recuperación de la literatura antigua, son muy escasas. Se debe llegar al siglo XVIII para encontrar, aunque parezca un contrasentido, un verdadero renacimiento del mundo clásico, especialmente en su vertiente literaria, que se extiende hasta la primera mitad de la centuria siguiente, especialmente en escritores que nacieron entre dos siglos y participaron de la corriente neoclásica. Entre esos escritores tuvo un lugar preferente el autor al que se va a prestar atención en las páginas siguientes, Graciliano Afonso Naranjo. Para una historia cabal de la pervivencia de los clásicos, tarea evidentemente inmensa, creo que son interesantes las aportaciones que hicieron muchos escritores particulares, desconocidos para la mayoría, incluso en su propio entorno, pero que lograron revivir el legado de los antiguos. Vayan ahora algunos datos, los más destacados, de este autor.

\section{Graciliano Afonso y los ClÁsicos grecolatinos}

La vida y la obra de Graciliano Afonso Naranjo, tras los trabajos de Alfonso Armas Ayala, algunos de ellos insustituibles por disponer de documentación que lamentablemente hoy no se ha podido encontrar ${ }^{7}$, ha empezado a tener interés tanto para los estudiosos de la Filología Hispánica ${ }^{8}$ como para los de

6. En concreto fueron tres las cátedras de Gramática establecidas: una, al amparo de la catedral de Las Palmas, en la isla de Gran Canaria; y otras dos, dependientes de los cabildos de La Laguna, en la isla de Tenerife, y Santa Cruz, en la isla de La Palma. Cf. el estudio de María Jesús Vera Cazorla, La ración de Gramática de la Catedral de Canarias, Las Palmas de Gran Canaria, Consejería de Educación, Cultura y Deportes, Col. «Fontes Ecclesiae Canariensis», 2003.

7. Cf. sobre todo, Alfonso Armas Ayala, Graciliano Afonso, un humanista canario, Tesis Doctoral inédita, Universidad de Madrid, 1953, resumida en Graciliano Afonso, un prerromántico español, La Laguna, Separata de Revista de Historia Canaria, 1963, del mismo autor, y en Graciliano Afonso: Prerromántico e ilustrado, Las Palmas de Gran Canaria, Ediciones del Cabildo Insular de Gran Canaria, 1993, del mismo autor.

8. Cf. sobre todo Antonio Becerra Bolańos : Graciliano Afonso: poeta, traductor y teórico de la literatura, Tesis Doctoral inédita, Universidad de Las Palmas de Gran Canaria, 2005 y, del mismo autor : La conformación de un canon: Graciliano Afonso, Las Palmas de Gran Canaria, Ediciones del Cabildo Insular de Gran Canaria, 2010. Se ha editado de este autor algunos textos. Véase, por orden cronológico, Andrés Sánchez Robayna, Museo Atlántico. Antología de la poesía canaria, Santa Cruz de Tenerife, Interinsular Canaria, 1983, pp. 92-98; Las bragas de San Grifón. Novela del abate Giambattista Casti traducida por el doctoral de Canarias, Graciliano Afonso, edición, prólogo y notas de Antonio Becerra Bolaños, Las Palmas de Gran Canaria, Ediciones del Cabildo Insular de Gran Canaria, 2003; Graciliano Afonso, Oda al Teide, el juicio de Dios o la reina Ico de Graciliano Afonso, Santa Cruz de Tenerife, Idea, 2004; Graciliano Afonso, El mar. Oda al Teide, edición, introducción y notas de Antonio Becerra Bolaños, Las Palmas de Gran Canaria, Archipliego, 2005; y Antología poética de Graciliano Afonso, introducción, edición y notas de 
la Filología Clásica9 en las tres últimas décadas.

Ello ha posibilitado que los datos esenciales sobre este escritor sean conocidos sobradamente en el ámbito literario de las islas sobre todo, sin menoscabo que el azar ayude a descubrir nuevos materiales que permitan ofrecer nueva información o apuntalar la existente.

En efecto, Graciliano Afonso nació en la villa de La Orotava en la isla de Tenerife en 1775, estudiando en el colegio que habían ocupado los jesuitas en dicho lugar. Aquí tuvo como profesor a José de Acosta, gran latinista y traductor de Virgilio y Horacio.

A los quince años se traslada a Las Palmas de Gran Canaria para estudiar en el Seminario Conciliar. Acrecentaría aquí sus conocimientos de las lenguas clásicas, sobre todo del latín, lengua importante en aquellos momentos para la carrera eclesiástica. $Y$ en este centro comenzó su carrera docente como profesor de Filosofía.

Obtiene luego el grado de licenciado en Derecho Civil y Canónico en la Universidad de Osuna, opositando a la Canonjía Doctoral en la Catedral de Canarias. Resulta elegido Diputado a Cortes en 1822, votando, desgraciadamente para él, a favor de la incapacidad de gobernar de Fernando VII. De resultas de ello, otra vez instaurado el absolutismo, se ve obligado a exiliarse en tierras americanas, concretamente en Venezuela y en la isla de Trinidad. Aquí, en su destierro americano, se refugia en la literatura (recordemos que algo parecido ocurrió con el poeta latino Ovidio), sobre todo en sus queridos autores clásicos, y aquí compondrá una gran parte de su obra literaria y humanística.

Tras promulgarse la amnistía, regresa a Las Palmas en 1838, después de pasar algunos meses en Puerto Rico. Ya en su tierra recupera su Canonjía doctoral e interviene sin descanso en los múltiples quehaceres que esa dignidad exigía. Participa también en la fundación del Colegio de San Agustín, ocupando la cátedra de Retórica y Poética durante algunos ańos. Muere en 1861, dejando una significativa labor como traductor de textos clásicos grecolatinos (también de otras lenguas) y como escritor de textos en vernáculo y latín ${ }^{10}$. Vieron la luz traducciones suyas de Anacreonte y Museo en Puerto Rico, en 1830, de la Eneida

Antonio Becerra Bolaños, Barcelona, Academia Canaria de la Lengua, col. «Biblioteca Manuel Padorno", 2007.

9. Cabe mencionar, como trabajo interdisciplinar que aúna la visión de filólogos clásicos e hispanos, Eugenio Padorno y Germán Santana Henríquez (eds.), Ilustración y pre-romanticismo canarios. Una revisión de la obra del Doctoral Graciliano Afonso (1775-1861), Las Palmas de Gran Canaria, Excmo. Ayuntamiento de Arucas - Fundación Mapfre Guanarteme de Arucas - Servicio de Publicaciones de la Universidad de Las Palmas, 2003.

10. Se describe la producción literaria del Doctoral Graciliano Afonso en Agustín Millares Carlo y Manuel Hernández Suárez, Biobibliografía de escritores canarios (Siglos XVI, XVII y XVIII), t. I, Las Palmas de Gran Canaria, El Museo Canario-C.S.I.C. Patronato "José María Quadrado» - Excmo. Cabildo Insular de Gran Canaria, 1975, pp. 40-64; y, concretamente, la producción humanística, en Francisco Salas Salgado, Humanistas canarios..., t. II, pp. 88-100. Cf. sobre el poema latino que se encuentra en la producción atribuida a Graciliano Afonso, copiada por Juan Padilla, Francisco Salas Salgado, «Acercamiento formal a un poema latino del siglo XIX en Canarias: el In promptu de Graciliano Afonso", Fortunatae, 2, 1991, pp. 297-312. 
y las Églogas de Virgilio, en Las Palmas, en 1854 y 1855, respectivamente ${ }^{11}$, y del Arte poética de Horacio, también en Las Palmas, en 1856. Además, en copia manuscrita de Juan Padilla conservamos otras traducciones de clásicos, como Píndaro, Sófocles, Homero o Esquilo ${ }^{12}$.

\section{La traducción de la ENEIDA de Virgilio por Graciliano Afonso:}

\section{EL PROCESO DE REDACCIÓN}

Sin embargo, de entre estas traducciones sobresale, sin duda, la realizada del poema épico virgiliano, cuyo título, transcrito de la portada, es La Eneida de Virgilio, traducida en verso endecasilabo por D. Graciliano Afonso, Doctoral de la Santa Iglesia Catedral de Canarias, año de 1853, Palmas de Gran Canaria, Imp. de M. Collina, Calle de la Carnicería, n. 1, 1854². Esta traducción, de marcada intención escolar, puesta de manifiesto por Afonso en la Advertencia preliminar que antecede a la misma, tuvo que ser ampliamente conocida entre aquellos que se acercaban a las aulas del Seminario o de los colegios de segunda enseñanza; por lo menos es la obra que más se conocía de este autor y en la que más empeño puso ${ }^{14}$.

He de confesar (nemini licet ignorare) que esta traducción ha estado muy presente en mi labor investigadora desde el año 1987, cuando, como Tesis de Licenciatura, hice un estudio de la misma, hasta hoy, en que ultimo una edición moderna ${ }^{15}$. Varios aspectos he estudiado de esta obra de Afonso. En este trabajo, tras la localización de diversos borradores de esta traducción previos a la edición de 1854, conservados en diversas instituciones de Las Palmas de Gran Canaria, concretamente en la Real Sociedad de Amigos del País y El

11. Según refiere Alfonso Armas Ayala (Graciliano Afonso, un prerromántico..., p. 398), había también traducido Afonso, junto con uno de sus alumnos predilectos, Amaranto Martínez de Escobar, las Geórgicas del mantuano.

12. Cf. para más datos, Alfonso Armas Ayala, Graciliano Afonso: Prerromántico..., pp. 99109; y Marcos Martínez Hernández, «Un anacreóntico canario: Graciliano Afonso», en Eugenio Padorno - Germán Santana Henríquez (eds.), op. cit., pp. 89-91.

13. Cf. Agustín Millares Carlo y Manuel Hernández Suárez, op. cit., p. 40.

14. Puede servir de ejemplo la necrológica publicada en el Boletín Oficial Eclesiástico de la Diócesis de Canarias y Tenerife, del día 20 de agosto de 1861. Allí se decía: «Escritor público y poeta ha legado a los amantes de las letras apreciables trabajos literarios, entre los cuales figuran la traducción en verso de la Eneida de Virgilio y del Arte Poética de Horacio con comentarios» (tomo la referencia en Agustín Millares Carlo y Manuel Hernández Suárez, op. cit., p. 74).

15. Cf. Francisco Salas Salgado, La versión de la Eneida de Graciliano Afonso: Aplicaciones del fenómeno de la traducción, Memoria de Licenciatura inédita, Universidad de La Laguna, 1987; «Sobre la versión de la Eneida de Graciliano Afonso», Revista de Filología de la Universidad de La Laguna, 8/9, 1989-1990, pp. 319-337; «Virgilio en Canarias: Versiones de su obra realizadas hasta el siglo XIX», en José M. a Maestre Maestre y Joaquín Pascual Barea (eds.), I Simposio de Humanismo y Pervivencia del Mundo Clásico, Cádiz, I.2, 1993, pp. 985-992; y «Reflexiones sobre la traducción del humanista canario Graciliano Afonso (La Orotava de Tenerife, 1775 - Las Palmas de Gran Canaria, 1861)», Cuadernos de Ilustración y Romanticismo, 11, 2003, pp. 49-65. 
Museo Canario, voy a intentar trazar los derroteros por los que pudo pasar el texto de la misma hasta su impresión última. Para ello conviene empezar por referir algunos datos que aparecen en la citada Advertencia preliminar.

\section{III.1 Las referencias del autor en la Advertencia preliminar}

Conviene señalar que esta traducción no escapa de las diversas consideraciones que se han establecido para la tradición escrita, no precisamente relacionadas con este siglo, caracterizado sobre todo por el espectacular avance de la prensa periódica, los adelantos técnicos, la aparición del escritor profesional y la ampliación y diversificación del público lector ${ }^{16}$. La precipitación en la entrega de los originales y en la impresión son algunos de los rasgos que definen las impresiones de la época y, seguramente, por lo que luego se dirá, deben aplicarse a esta traducción de la Eneida. A ello habría que añadir el hecho, no raro, de que el autor, por determinadas circunstancias, no se ocupara personalmente de la corrección de pruebas o necesitara ayuda para ello.

Contamos para el conocimiento de los estadios previos del texto impreso de esta traducción de Virgilio con los datos del propio Graciliano, en esa especie de admonitio auctoris que es la Advertencia preliminar. A pesar de ser un párrafo de cierta extensión, resulta del todo imprescindible transcribirlo por los datos que aquí se ofrecen. Dice así:

En el año de 1838 traje de América, donde permanecí 18 años emigrado por la cáusa de la libertad, una traduccion de la Eneida en prosa con notas, para la instruccion de la juventud canaria. Pero en aquel tiempo, fué imposible darla á la prensa, porque entonces ni podia haber clérigos, ni se pagaba al clero existente, que iba concluyendo como por inanición. Llegó el momento del actual concordato, y cuando creí que con los nuevos nombramientos sobrára tiempo para verificar la impresion dicha, hallé que al dar un abrazo de enhorabuena al Dean nuevamente nombrado Dr. D. Nicolás Calzadilla, quedó admirado de verme en sana salud; y mucho mas, noticioso de cuanto habia trabajado durante la miseria de operarios en la Iglesia Catedral, y durante el Cólera. Yo habia quedado estacionario en mi destino de Doctoral (fácil es adivinar la cáusa habiendo sido diputado en las Córtes de 1822 y comprendido en el infame nombre de amnistía), porque el Gobierno se hallaba persuadido de que un ataque de perlesía me tenía postrado en cama poco menos que insensato. Pero yo, que como tal Diputado de aquella época, jamás habia vestido el escapulario de pretendiente, oí esto con mi ordinaria indiferencia, contentándome con desmentir con mi conducta las palabras falaces y mentidas de los Caritativos Eclesiásticos que me hacían la guerra á salva mano. Entonces recurrí á mi auxilio ordinario de la lectura, y al cumplimiento de mi obligacion; y curioso de ver el estado de mi Eneida prosáica, que ocurrió en 25 de junio de 1853, despues de ver dos o tres hojas, mi primer pensamiento fué: Veamos si anda la noria el perro. Palabras de mi paisano Iriarte en su fábula 29, El gozque y el macho de noria. Porque, en efecto, 78 años no anunciaban ciertamente mas que la fuerza de un miserable gozquejo, para mover la pesada rueda de una noria. Y por

16. Me he servido fundamentalmente del manual de Alberto Blecua, Manual de critica textual, Madrid, Castalia, 1983, pp. 227-232, y del clásico de Paul Maas, Critica del Testo, trad. italiana di Nello Martinelli, Firenze, F. Le Monnier, 1966. 
diversion, ó mas bien, para burlarme de mí mismo, puse en verso el libro primero, y leido á mi amigo el Licenciado D. Bartolomé Martínez y noble familia, aficionada á la poesía y literatura; me persuadieron, que aunque no fuese para imprimirla, continuase la repetida traduccion. El resultado fue, que por consecuencia de mi carácter, emprendí con tanto ardor mi tarea, que á pesar de una molesta hinchazon de pies, el 24 de octubre del mismo ańo quedó concluida enteramente la obra, sin que me aterrase otro espectaculo que el de la imágen de la muerte, compañera inseparable de la vejez ${ }^{17}$.

Visto lo anterior, se puede considerar que esta traducción de la Eneida tuvo en sus comienzos dos fases. Una primera sería la traducción en prosa con notas que realizara el autor durante el destierro en tierras americanas, la cual había traído en su regreso a las Islas. El periodo de realización de esta traducción comprende un intervalo de quince años y no dieciocho, como se indica en esta "Advertencia preliminar» (son números que se prestan a la confusión), a saber, desde 1823 a 1838 . Una segunda fase corresponde al momento en que se pasó esta traducción de prosa a verso, y cuya realización, ya en las Islas, hubo de hacerse de junio a octubre de 1853, es decir, en apenas cuatro meses (luego se verá por los testimonios manuscritos que he podido encontrar que fue una etapa de intensa actividad). Todo ello, como se supone, se materializó en el texto impreso de 1854. Tratemos a continuación de los materiales que acreditan dichas etapas.

\section{III.2 La versión en prosa de la Eneida: algunos testimonios}

Conviene indicar de antemano que, a pesar de las continuas pesquisas que he realizado, no he podido localizar el texto de esta traducción en prosa. Desconozco si se encuentra en el fondo que supuestamente contiene la obra del Doctoral canario o se ha perdido definitivamente ${ }^{18}$. Sin embargo, existen algunos documentos que podrían tener relación con este primer estadio en la transmisión del texto.

Uno de estos documentos es un manuscrito que lleva el título Notas del Libro $4 .^{\circ} \|$ de la $\|$ Eneida, que se encuentra en El Museo Canario de Las Palmas. Consta de 16 hojas sin paginar y contiene un total de 81 anotaciones que ocupan 14 páginas por ambas $\operatorname{caras}^{19}$. Cada una de las anotaciones que aparece en este manuscrito lleva un enunciado que se refiere al texto de la traducción. La relación con el texto de la versión en prosa se deduce del hecho que estas referencias no se corresponden en ningún momento con el texto en verso.

17. Cf. Graciliano Afonso, «Advertencia preliminar», La Eneida de Virgilio, traducida en verso endecasilabo por D. Graciliano Afonso, Doctoral de la Santa Iglesia Catedral de Canarias, Palmas de Gran Canaria, imp. de M. Collina, 1854, pp. III-V. Advierto que, en las citas del texto de la edición y de los manuscritos, respeto la ortografía y la puntuación.

18. Se trata del Archivo Mesa y López, hoy en manos particulares, donde puede que existan materiales al respecto.

19. Las Palmas de Gran Canaria, El Museo Canario, "Col. documental Gregorio Chil y Naranjo». Cf. Agustín Millares Carlo - Manuel Hernández Suárez, op. cit., t. I., pp. 41-42; y Francisco Salas Salgado, Humanistas canarios..., t. II, p. 99. 
Viene a apoyar esta suposición otro texto, mecanografiado, que he localizado entre los papeles pertenecientes al profesor Alfonso Armas Ayala.

$\mathrm{El}$ archivo que perteneció a este investigador de la literatura canaria y gran conocedor de la obra de Graciliano Afonso se encuentra en la biblioteca de la Casa de Colón en Las Palmas de Gran Canaria. Entre los papeles que le pertenecían se encuentra una versión mecanografiada sin paginar de la que fue su Tesis Doctoral con correcciones a mano, seguramente suyas, realizadas posiblemente con la intención de publicar dicho trabajo ${ }^{20}$. El capítulo VII, titulado El traductor, que no apareció luego publicado, lleva como Apéndice III bis la traducción en prosa de parte del libro $\mathrm{V}$ y unas notas, iguales formalmente a la del manuscrito, es decir, con un encabezado numerado correlativamente que se corresponde a unas llamadas previas en el texto de la traducción hechas entre paréntesis. No se nos escapa que A. Armas Ayala pudo tener acceso a esta versión en prosa, y a ella debe pertenecer la copia que incluye en este apéndice.

Además, las notas que siguen a la traducción de este libro quinto permiten conocer datos sobre otras traducciones que conocía nuestro autor y de las que, de hecho, hizo uso. Al comienzo se lee un comentario previo donde se menciona el nombre de Detule. Léase así:

Antes de empezar con las notas, no será fuera de propósito, para la instrucción del joven lector, dar un ligero extracto de lo que con tanta propiedad observa Detule (sic) sobre las excelencias de este libro $5 .^{\circ}$. Este libro $5 .^{\circ}$ es acaso el más trabajado de toda le Eneidas (sic) y se nota en todo el cuidado y el esmero del traductor. En los precedentes y siguientes hay cuadros que cautivan la imaginación y la sensibilidad. El naufragio de los Troyanos, su llegada a Cartago, su permanencia en aquella ciudad que con el tiempo sería la terrible enemiga de Roma, capital de su reino futuro y de todo el mundo; la narración de sus aventuras, la ruina del imperio de Príamo; el encuentro de Andrómaca y Enea, en el Epiro; los amores y trágico fin de Dido: su descenso a los infiernos; la futura grandeza del pueblo Romano anunciada en visiones proféticas; este largo prenado (sic) que debía dar a luz un imperio que empezó en las cabañas del Lacio, y no debía tener límites en gloria y duración; todo esto daba materia para despertar el genio de la Epopeya, y continuar la ascensión y el interés de los lectores que se hacían Romanos en su lectura. Pero los juegos del lib. 5. ${ }^{\circ}$ no están enlazados con aquellos grandes acontecimientos o de una gran pasión, él sólo podría unir los fuegos por medio de la versificación más perfecta. Nunca hizo brillar Virgilio su inmenso poético y las dificultades que le acompañan $[\ldots]$.

Detrás de este nombre, evidente error en la transcripción realizada por Armas Ayala, se esconde la figura de Jacques Delille, de notoria fama entre los ilustrados canarios $^{21}$, de quien toma las referencias y a quien por momentos sigue casi de forma literal. Compárese, así el comentario anterior con el siguiente pasaje de Delille:

20. Cf. nota 7 del presente trabajo. Alfonso Armas Ayala (Graciliano Afonso: Prerromántico..., p. 107) reproduce el inicio de este libro $V$, y sus palabras dan a entender que forma parte de la traducción en prosa realizada en tierras americanas.

21. Como ejemplo, el ilustre polígrafo José de Viera tenía pensado realizar la traducción del libro primero de las Geórgicas de Virgilio, teniendo a la vista el texto latino y la traducción del abate Delille. Cf. Francisco Salas Salgado, Humanistas canarios..., t. I, p. 269. 
Ce cinquième livre est peut-être celui dont Virgile a travaillé tous les détails avec le plus d'art et de soin. Dans ceux qui précèdent et dans ceux qui suivent, l'imagination et l'ame sont intéressées par des tableaux tour-à-tour sublimes ou touchants. Le naufrage des Troyens, leur arrivée à Carthage, leur séjour dans cette ville qui sélève pour être un jour l'ennemie de celle quiils vont fonder en Italie, le récit de leurs longs malheurs, l'incendie de Troie et la chute de l'empire de Priam, la rencontre d'Énée et d'Andromaque en Épire, les amours et la fin tragique de Didon, la descente aux enfers, les grandeurs futures du peuple romain, annoncées de loin dans des visions prophétiques; enfin, ce long enfantement d'un empire qui doit soumettre le monde, et qui commence dans les cabanes du Latium: tous ces sujets ont dî facilement élever le génie de l'épopée, et sont faits pour plaire à tous les lecteurs. Mais ici le poëte n'a peint que des jeux; il n'était plus soutenu par l'intérêt des grands événements ou d'une grande passion, et dès lors il ne pouvait attacher qu'à l'aide d'une versification parfaite. Aussi n'a-t-il jamis porté plus loin le talent de la difficulté vaincue et les effets du style pittoresque ${ }^{22}$.

Otro tanto sucede con las anotaciones manuscritas de Afonso para el libro cuarto, de semejanza apreciable con las realizadas para el mismo libro por el poeta francés. Algunas de estas notas reproducen fielmente el texto de Delille, como ocurre, con la anotación 6 de Graciliano y la 9 del francés, referida al verso agnosco veteris vestigia flammae; otras, aunque no exista una imitación, también toman como modelo la traducción del autor francés. Por ejemplo, véase la nota 27 de este libro cuarto:

\section{(27.) En oro y purpura $\&^{\mathrm{a}}$}

Stat sonipes et frena ferox spumantia mandit

Representa un caballo brioso, pero tranquilo y dócil, enjaezado digno de una Reina.

Tandem progreditur. Ya nada detiene la marcha: es privilegio de los Reyes no esperar por nadie, por poco no me hacen esperar decia el Luis Catorce.

Si hubiera sido Rey el soberano de Cartago, Virgilio no se detendria tanto en describir el traje de la Jóven Reina: pero era muger reina y amante, y agradar era su intento y el poeta debia manifestarlo. Que riqueza, que magnificencia en el traje de cazadora. Eneas y los Troyanos hacen el primer papel. Muchos creen, y no sin causa que la comparacion de Eneas con Apolo no es esacta (sic) cual convenia, pero estal (sic) la harmonia poetica, tan acabado este aparato de cacería, que preciso es olvidar que

Apolo del Xanto volviendo á Delos no se asemejaba al cazador Eneas, aunque Venus le habia adornado con toda las gracias de la belleza.

La caceria con harmonia (espacio) silabas largas y breves consonantes y vocales, es el encanto de esta descripcion. No se bien la de Ascanio, á quien volveremos á ver tan bello en los juegos del Lib. $5^{\circ}$ y nos hará recordar los versos del Maestro[... $]^{23}$.

\section{Y confróntese con el texto de Delille:}

Stat sonipes, ac frena ferox spumantia mandit.

Ce vers exprime admirablement un cheval bien dressé, qui réunit ensemble l'ardeur et la docilité. On y trouve d'ailleurs une magie d'harmonie, qui fait qu'on croit entendre l'action d'un coursier fougueux rongeant son frein d'impatience.

22. L'Enéide, traduite en vers français, par J. Delille, seconde édition, revue et corrigée, avec les variantes. Tome deuxième, À Paris, chez Michaud Frères, libraires, MDCCCXIII, pp. 224-225.

23. [G. Afonso Naranjo], Notas del Lib. $4^{\circ}{ }^{\circ}$ de la "Eneida», [s.a.], [s.l.], ff. 5ำ-6 
Tandem progreditur exprime parfaitemente la longue attente occasionnée par le retard de la reine, et le plaisir que fait naître sa présence. Un des priviléges de la grandeur est de ne pas attendre, et d'être attendu. Louis XIV, arrivant en même temps que sa voiture au pied du grand escalier de Versailles, dit, en se retournant vers son grand-écuyer: J'ai failli attendre. Ce mot exprime vivemente le sentiment quil avait de l'élévation de son rang et du respect qui lui était dû.

Si le souverain de Carthage avait été un homme, Virgile ne se serait peut-être pas arrêté à décrire son costume; mais une jeune reine, mais une amante interessée à plaire, ne devait rien avoir oublié de ce qui pouvait y contribuer. Le poète lui prête tout le goût et toute la magnificence dont une toilette de chasse est susceptible. Il est tout simple que, dans ce jour, les Troyens et leur chef jouent un des premier rôles. La comparaison d'Enée avec Apollon, si elle n'est pas d'une grande exactitude, est de la plus belle poésie. C'est toujours aux dieux qu'Homère et Virgile comparent les hommes qu'ils veulent faire valoir. Dans la suite de cette description, le lieu de la scène, les chasseurs, les animaux poursuivis, sont peints avec tout le mouvement et toute la vérité nécessaires. Pour faire partir ces animaux sauvages, Virgile attend, avec, raison, que la chasse soit parvenue dans les taillis les plus épais et les lieux les plus impraticables; alors, par un mélange heureux de syllabes brèves et longues, par la cadence et la coupe des vers, il nous fait entendre les sauts, les bonds impétueux, et la fuite précipitée des daims, des chevreuils et des chamois chassés précipitamment de leurs retraites. Nous avons remarqué ailleurs comment il a peint, dans la personne d'Ascagne, l'ardeur et l'émulation que mettent les jeunes gens dans ces sortes d'exercice ${ }^{24}$.

Es, por tanto, el texto de la traducción de la Eneida del francés Jacques Delille el que sirvió de modelo para esta traducción en prosa. Sin embargo, no se puede decir lo mismo de la traducción en verso; una comparación entre ambas hace ver lo contrario, si bien hay que considerar que el texto de Delille pudo seguir siendo útil a nuestro traductor. Véase, del mismo libro IV, la traducción que ambos hacen de los cinco versos iniciales de Virgilio:

\author{
J. Delille, IV, 1-6 \\ La reine cependant, atteinte au fond de l'ame, \\ Nourrit d'un feu secret la dévorante flamme: \\ Le héros, sa beauté, son grand nom, sa valeur, \\ Restent profondément imprimés dans son cour, \\ La voix d'Énée encor résonne à son oreille, \\ Et sa brûlante nuit n'est qu'une longue veille \\ G. Afonso, IV, 1-9 \\ De la pena mas cruel la reina herido \\ Siente su puro y amoroso seno, \\ $\mathrm{Y}$ al corazon profundamente abrasa \\ De oculta llama inestinguible fuego. \\ De Eneas el valor y la grandeza, \\ Su semblante, su origen y su acento, \\ Todo junto en su mente está grabado. \\ Ni su pena calmara el dulce sueńo, \\ Que amorosa inquietud el pecho agita.
}

24. L'Enéide, traduite ..., pp. 108-109. 


\section{3 La traducción en verso: tradición textual}

El texto de la traducción de la Eneida se nos ha transmitido a través de dos copias manuscritas, sobre las que existen pocas referencias, especialmente de una de ellas, como se verá, y que deben ser previas a dicha edición de 1854 . Conviene observar que dada la escasez de datos que sobre las mismas poseo cualquier consideración que se haga aquí se debe entender en el terreno de la hipótesis. Una de las copias, de casi la totalidad de la traducción, se encuentra en el archivo de la Real Sociedad Económica de Amigos del País de Las Palmas de Gran Canaria, a la cual nombramos $E$, y otra, sólo de dos libros, se localiza en El Museo Canario de esta ciudad insular, que designamos $M$. Se intentará en las siguientes páginas determinar el proceso previo de composición de esta traducción a través de la comparación de estos manuscritos estableciendo la posible filiación de los mismos y su relación con el texto impreso.

\section{III.3.1 Los manuscritos de la Real Sociedad Económica de Amigos del País}

Se conservan en esta institución, nacida en el seno de la Ilustración, los doce libros manuscritos de la traducción de la Eneida en un estado de conservación aceptable.

La referencia a este manuscrito en el índice de la biblioteca de esta institución es solo la siguiente: «Serie 6, Manuscritos, legajo 1». No hay mención de autor, de poseedor, ni ninguna otra indicación sobre su procedencia. Cada libro aparece escrito en varios pliegos, constando de portada, donde va la referencia al libro de la Eneida que se traduce, y el texto de la traducción. Estos pliegos van numerados en el recto de la primera hoja y cosidos posteriormente, siendo su tamaño de 235 x $170 \mathrm{~mm}$., por lo general. Sólo el libro quinto está incompleto, faltando los primeros 312 versos.

El manuscrito es apógrafo, la letra no es de Graciliano Afonso, con la intervención en algunos momentos de dos manos. En comparación con otros manuscritos que he podido revisar, la letra de una de las manos se asemeja a la de una carta que se encuentra pegada al primero de los tomos de la edición de esta traducción de la Eneida que se localiza en el Fondo Maffiotte de El Museo Canario, aunque no se sabe quién pudo ser su autor. En esta carta se puede observar claramente la firma, al final, de Graciliano Afonso, de trazo tembloroso, diferente a la del texto, escrito en una elegante cursiva.

Este manuscrito está lleno de correcciones, algunas de las cuales parecen haber sido hechas en el momento de la copia, pero otras fueron realizadas posteriormente, perceptible esto por la diferencia de color de la tinta. Otras correcciones se hicieron también a lápiz. En este trabajo de lima muchas veces se tacha el texto sin añadir nada, y en otros momentos se añaden versos en los márgenes o en hojitas pegadas. Aparecen también en los márgenes reclamos cuyo fin es llamar la atención sobre determinados añadidos. 
Asimismo el proceso de redacción de esta traducción se puede seguir a través de la fecha que aparece en algunos de estos libros en el margen izquierdo superior del recto de la primera hoja ${ }^{25}$.

Un aspecto que habría que considerar es que el texto de este manuscrito contenga -sobre todo al ser un borrador- la versión en verso que realizara Afonso de aquella traducción en prosa que había traído de América. Además, al ser objeto de una revisión, habría que admitir una intervención voluntaria del copista, quien, con esa labor de lima, altera el texto con un propósito determinado. Tampoco sabemos si el texto fue dictado por el autor o es copia de un supuesto autógrafo del propio Graciliano Afonso (este segundo supuesto explicaría que la mayor parte de los nombres propios que aparecen no se transcriban en principio correctamente y tengan que ser revisados).

Atendiendo a lo anterior podemos establecer una diferenciación en el proceso de escritura de este borrador: un primer momento corresponde a una primera copia, que llamaremos $E_{1}$, el cual, como dije antes, puede ser el paso de la traducción de la Eneida de prosa a verso; un segundo momento, de relectura de este manuscrito, corresponde a las correcciones, que denominamos $E_{2}$.

A este respecto, en $E_{2}$ se pueden distinguir las siguientes enmiendas ${ }^{26}$ :

a) Supresiones, esto es, se eliminan fonemas o palabras, simplemente tachándolas:

\author{
I, 50 \\ Y yo hermana y esposa del supremo \\ Sumpremo $\mathrm{E}_{1}$ : supremo $\mathrm{E}_{2}$ \\ II, 4 \\ Yo las viera y tambien las alcanzara \\ también yo $\mathrm{E}_{1}$ : también $\mathrm{E}_{2}$ \\ III, 17 \\ Y obedeciendo ciegos á su mando \\ $Y$ del él obedeciendo $\mathrm{E}_{1}:$ obedeciendo $\mathrm{E}_{2}$ \\ XI, 314 \\ El principe Troyano y Tárcon manda \\ Tracon $\mathrm{E}_{1}$ : Tarcon $\mathrm{E}_{2}$
}

b) Adiciones, es decir, se añaden sílabas y palabras que en $E_{1}$ no se pusieron, debido seguramente a la prisa con que se hizo la copia, pero también versos enteros, tanto encima como en los márgenes del manuscrito:

III, 68

Yo para ti; si sangre ves brotando

si brotando $\mathrm{E}_{1}$ : si sangre ves brotando $\mathrm{E}_{2}$

25. Así el libro $3^{\circ}$ lleva la referencia "Julio 23 de 1853 ", el libro 6º "Agosto 15 de 1853», el

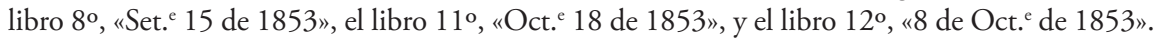

26. Reproduzco primero, en redonda, el texto de la edición, con indicación de libro y verso, y luego, en cursiva, el del manuscrito. 
III, 299

$\mathrm{Y}$ las manos al cielo dirigiendo

digiendo $\mathrm{E}_{1}$ : dirigiendo $\mathrm{E}_{2}$

VI, 64

Dáte prisa y al punto sean tu ofrenda

sea $\mathrm{E}_{1}: \operatorname{sean} \mathrm{E}_{2}$

c) Alteración del orden, normalmente de palabras, como en:

\section{V, 323}

Ya no aspira Mnesteo á la primera

Mnesteo no aspira $\mathrm{E}_{1}$ : no aspira Mnestheo $\mathrm{E}_{2}$

d) Sustituciones:

I, 74

Devore el Ponto, y destrozados cuerpos

I, 74 los sangrientos $\mathrm{E}_{1}$ : destrozados $\mathrm{E}_{2}$

VII, 34

Y los lobos ahullan; todos víctimas

los lobos abullaban $\mathrm{E}_{1}$ : los lobos ahúllan $\mathrm{E}_{2}$ (metri causa)

Al margen de estas peculiaridades, el carácter de texto no definitivo de $E$ queda reflejado en la cantidad de lecturas que lo separan de la edición, por lo que hay que considerar que el proceso de escritura continuó, existiendo muy probablemente otras copias. Ejemplo de lecturas divergentes son (y ello passim):

I, 438

De ellos sus ojos y un olor sabeo

De ellos sus] Sus brillantes E

II, 292

serían destruidos (quiera Palas

Serian] Seriais E

III, 114

Confiar el buque al líquido mar ancho

Confiar el buque] Confiarse alguno al $\mathrm{E}$

IV, 375

Los campos que el Destino le promete

Los] $Y \mathrm{E}$

V, 508

Y este timbre de honor, alcanzen cuantos

alcanzen cuantos] los tendrán cuantos combatan $\mathrm{E}$ 
VI, 139

El Dios, remueve su espumosa boca

remueve] y agita $\mathrm{E}$

VII, 1017

Y se aumenta el temor que le inspirara

$Y$ se aumenta el temor] Ni el temor detener E

VIII, 43

Del líquido movible el que reflecta

movible] cristal $\mathrm{E}$

IX, 4

Del elevado Olimpo, cuando él fuera

Del elevado Olimpo] De lo alto del cielo E

$\mathrm{X}, 213$

De oro entonces deja, y las Deidades

Deidades] los Dioses E

XI, 693

De la Nacion Sabina, hoy la guerra

Sabina] Latina E

XII, 405

Mas cambian su pensar los que suspiran

Mas cambian] Y mudan E

\section{III.3.2. Los manuscritos de El Museo Canario}

Ya Agustín Millares Carlo y Manuel Hernández Suárez ${ }^{27}$ advirtieron de la existencia de unos manuscritos de esta versión en endecasílabos de la Eneida que se encontraban en esta institución, que acompañaron de una descripción física de los mismos. En concreto el libro $2^{\circ}$, el $4^{\circ}$ y unas notas independientes, el $6^{\circ}$, el $7^{\circ}$, el $8^{\circ}$, el $9^{\circ}$, el $10^{\circ}$, el $11^{\circ}$ y el $12^{\circ}$. De estos manuscritos sólo se conservan el libro $2^{\circ}$ y el $4^{\circ}$, en una caja que en el lomo tiene la leyenda «Poesías // G. Afonso». Las medidas aproximadas son 160 × $215 \mathrm{~mm}$., y sus otras características físicas concuerdan con las descritas por Millares y Hernández.

En concreto, la portada del manuscrito $2^{\circ}$ lleva escrito a lápiz «Traducción de D. G. Afonso» de letra de Aurina Rodríguez Galindo, bibliotecaria que fue de esta institución hasta 1981. Los versos están numerados, también a lápiz, de cinco en cinco. El proceso de redacción por la diferencia de color de la tinta parece que tuvo diversos momentos ${ }^{28}$. La mano de muchas de las

27. Cf. Agustín Millares Carlo - Manuel Hernández Suárez, op. cit., t. I., pp. 41-42.

28. Se distingue, así, un primer momento hasta el verso 108; un segundo, en los vv. 109-306; un tercer momento, en los vv. 307-541; un cuarto, en vv. 541-914, y un quinto momento, en vv. 915-1275. 
correcciones no parece la misma que la del texto, lo cual hace suponer que estas correcciones fueran hechas a posteriori; otras, en cambio, parece que se hicieron en el momento de la copia.

Por su parte el manuscrito del libro $4^{\circ}$ lleva también en la portada a lápiz la referencia de Aurina Rodríguez Galindo. También están numerados a lápiz los versos de cinco en cinco. Aquí la mano de las correcciones es totalmente ajena a la mano que realiza la redacción. También tuvo que realizarse el proceso de corrección en diversas etapas ${ }^{29}$.

Una primera cuestión a tratar es la relación que estos dos manuscritos (deben considerarse todavía borradores y no textos definitivos) guardan con $E$.

A este respecto, se aprecia que el texto de $M$ reproduce el texto de $E$ (tanto de $E_{1}$ como de $E_{2}$ ). Esta es la condición principal de $M$, la de ser copia de un borrador $(E)$ que por momentos se presentaba ilegible, sin intención de realizar cambios (es difícil lo contrario). Ello puede observarse:

a) En los lugares donde el copista de este manuscrito reproduce, incluso, las correcciones de $E$. Valgan como ejemplos los siguientes, en los que $M$ copia las mismas tachaduras que existen en el manuscrito de la Económica:

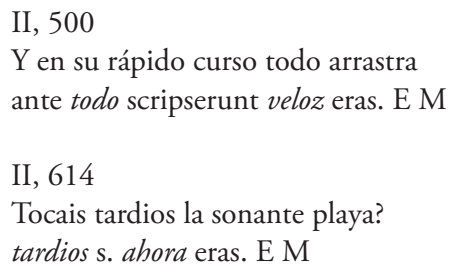

b) En los añadidos posteriores que hay en $M$, errores de omisión del copista seguramente por la rapidez con que el texto fue escrito, y que corresponden a lecturas que había en $E$. Es frecuente esto en palabras con poca entidad gráfica, como conjunciones, artículos o pronombres, $v . g r$ :

II, 182

Que de Troya llegaron á las playas

des. 1. M

IV, 387

No le inflama el deseo de su gloria

inflama E: inflama M (-l-s.l.)

aunque, también podemos encontrar otras categorías gramaticales, caso de:

IV, 196

Con Eneas salir se habia propuesto

salir s. 1. M

29. Se advierten por el diferente color de la tinta varias fases en el proceso de redacción: una primera ocupa los vv. 1-741; la segunda tirada, los vv. 742-1134; y un tercer momento, los vv. $1135-1215$. 
c) Por último, en determinadas lecturas que invitan a considerar una intención ¿posterior? de corregir el texto de $E$, aunque al final se opte por dejarlo. Ejemplos de ellos en:

\author{
II, 474 \\ Mas sus dioses Penates y su patria \\ sus $\mathrm{E}$ : sus s. los eras. $\mathrm{M}$ \\ II, 482 \\ Que ardiera eternamente ante las aras \\ eternamente s. enteramente eras. M
}

Esta última caracterización de $M$ (que también pudiera entenderse como error del copista en el proceso de lectura, memorización, dictado y transcripción) se torna en evidencia cuando nos damos cuenta de que al posible carácter de puesta en limpio de este manuscrito se suma el contener correcciones al texto de $E$, coincidiendo de esta manera $M$ con la edición. De esta manera, por un lado $M$ corrige lecturas de $E$, caso de:

II, 81

Y él astuto bien lejos de oponerse

pérfido E: astuto (astuto s. pérfido eras.) M

II, 342

Le ciñen con cerúleas escamas

ciñen su cuello con cerúlea escama E: le ciñen su cuerpo (su cuerpo eras.) con ceruleas escamas M

En otros lugares, las correcciones de $M$ son añadidos ${ }^{30}$. Así en:

II, 985

A Juno mira que en las puertas Sceas

que s.l. M: om. E

Sin embargo, en $M$ ya existen lecturas diferentes que invitan a considerar la hipótesis anteriormente planteada. Ejemplos de ello en:

II, 200

Pero instó Ulises y por fin declara

insta $\mathrm{E}$ : instó $\mathrm{M}$

II, 257

Las guardias, y tambien la casta vírgen

$y$ de E: $y$ también $\mathrm{M}$

30. La mayor parte de estas adiciones se hizo con posterioridad, cuando se empezó a corregir el texto. Ello es fácilmente apreciable por el diferente color de la tinta. Así, por ejemplo, en IV, 324: Las noches pasan del cansado invierno, el texto de $E$ trae pasa y el de $M$ pasan, y se nota que la - $n$ final de $M$, es añadido posterior por la diferencia en la tinta y por el trazo más grueso. 
IV, 477

Manda equipar la escuadra con presteza

armada E: escuadra $\mathrm{M}$

Con todo, a pesar del avance que supone el texto de $M$ en relación con el texto definitivo que tenemos en la edición, existió un limae labor posterior, documentado en determinadas variantes donde $E$ y $M$ coinciden frente a la lectura diferente que encontramos en la edición. Véanse los siguientes ejemplos:

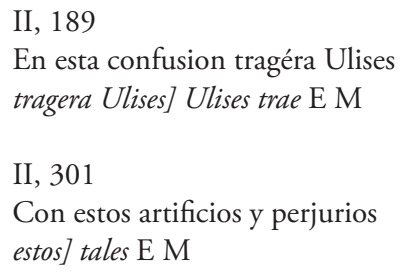

II, 306

$\mathrm{Ni}$ el fuerte Aquiles, ni diez años de armas

Achiles E M

\section{IV, 220}

Muy numerosa con su ardor inquietos

inquieto $\mathrm{E} \mathrm{M}$

IV, 245-246

Que le sirvió de cuna, con las danzas

De los alegres coros de los pueblos

danzas] fiestas $\mathrm{E} \mathrm{M} \mid$ alegres] danzantes $\mathrm{E} \mathrm{M}$

IV, 351

En las nubes rodando sin designio

rodando] rodaron $\mathrm{E} \mathrm{M}$

IV, 558

No existiera jamás solo un momento

jamás solo un momento] en verdad algun momento $\mathrm{E} M$

\section{III.3.3 La edición de 1854}

Este texto, que suponemos definitivo y que no se ha localizado, es el que pudo servir de modelo para realizar la impresión. La edición de esta traducción se dividió en dos tomos, encuadernados en un solo volumen -aunque también se encuentra en dos- con paginación independiente.

El primer tomo consta de VIII páginas preliminares, una "Advertencia al lector» fechada el 2 de agosto de 1854 en Las Palmas, y de 233 páginas en las que se encuentra el texto de la traducción de los seis primero libros de la Eneida.

El segundo tomo lleva idéntica portada. Consta de 278 páginas, donde aparecen el texto de la traducción de los últimos seis libros del poema épico, 
además de «Rectificaciones» de los tomos primero y segundo y de «Erratas» de ambos tomos. Esto último hace que se considere que hubo todavía entonces una labor correctora, seguramente antes de terminar la impresión de la obra ${ }^{31}$. Así aparecen:

\section{RECTIFICACIONES DEL TOMO PRIMERO}

En la pág. 5a verso 17 donde dice: Del abismo despojos revolviendo; faltan los cuatro versos siguientes:

$$
\begin{aligned}
& Y \text { raros aparecen en el vasto } \\
& \text { Piélago, y nadan armas de guerreros, } \\
& \text { Con las pinturas, y maderos rotos, } \\
& Y \text { las riquezas del Troyano imperio. }
\end{aligned}
$$

$\begin{array}{llll}\text { Pág. } & \text { Ver. } & \text { Dice } & \text { Léase } \\ 30 & 15 & \text { os harán } & \text { osarán } \\ 55 & 14 & \text { pelea á espada } & \text { Pelea espada } \\ 100 & 16 & \text { es, el que } & \text { crueles, que } \\ \text { Id. } & 17 & \text { Ovejas. } & \text { ovejas, } \\ 112 & 5 & \text { y con astucia } & \text { y de su astucia } \\ 124 & 27 & \text { Compasiva } & \text { compasivos, } \\ 145 & 23 & \text { alguna tierra amiga, } & \text { algun pais amigo; } \\ 223 & 30 & \text { y de donde } & \text { ydonde }\end{array}$

RECTIFICACIONES DEL TOMO SEGUNDO

$\begin{array}{llll}\text { Pag. } & \text { Ver. } & \text { Dice } & \text { Léase } \\ 30 & 31 & \text { obliga. } & \text { obliga, } \\ 33 & 25 & \text { Alpunto } & \text { Alpuerto } \\ 40 & 8 & \text { Otras tantas } & \text { Otra tanta } \\ 41 & 32 & \text { en paz } & \text { en pos } \\ 43 & 1 & \text { Son } & \text { con } \\ 191 & 31 & \text { Agamenon rey } & \text { Agamenon }\end{array}$

ERRATAS DEL TOMO PRIMERO

$\begin{array}{llll}\text { Pag. } & \text { Ver. } & \text { Dice } & \text { Léase } \\ 20 & 4 & \text { mirase aun } & \text { miran aun } \\ 52 & 32 & \text { Carro } & \text { el carro } \\ 86 & 16 & \text { de Ceres el vástago } & \text { de Creúsa el vástago } \\ 95 & 18 & \text { Propicio } & \text { propicios } \\ 112 & 26 & \text { Trensa } & \text { trenza } \\ 139 & 24 & \text { Armada } & \text { amada } \\ 210 & 25 & \text { Con ella } & \text { Con ellas }\end{array}$

31. Aparecen en las páginas 277 y 278 del segundo tomo. Valga apuntar que en la edición moderna de este texto que estoy preparando son otras muchas las erratas que he podido localizar, seguramente debidas a despistes del impresor, y que escaparon también al ojo crítico del corrector. 
ERRATAS DEL TOMO SEGUNDO

$\begin{array}{llll}\text { Pag. } & \text { Ver. } & \text { Dice } & \text { Léase } \\ 6 & 24 & \text { ihijo mio! } & \text { ¡O hijo mí! } \\ 42 & 23 & \text { Pera. } & \text { Para } \\ 44 & 13 & \text { canastilla; } & \text { canastilla } \\ I d . & 14 & \text { Minerva. } & \text { Minerva; } \\ I d . & 26 & \text { Como al oro } & \text { Como de oro } \\ 88 & 16 & E & e ́ \\ 101 & 23 & \text { Sexo } & \text { Seso } \\ 191 & 18 & \text { Cubea } & \text { de Eubea } \\ 219 & 29 & (=30) \text { silvando } & \text { silbando }\end{array}$

\section{Consideraciones finales}

Visto lo anterior, podemos extraer algunas conclusiones sobre el proceso de redacción de esta traducción, que, a pesar de ser corto en el tiempo, fue bastante laborioso.

Lo primero que habría que considerar es que, al no ser definitivos los manuscritos que conservan el texto, el editor moderno no puede realizar un verdadero trabajo de crítica textual (considérese también aquí las carencias de los mismos en cuanto a versos o ejemplares).

La tradición manuscrita en la obra que tratamos debe hacerse a través de borradores que contienen correcciones abundantes, sobre todo algunos de ellos, interesantes, eso sí, para distinguir las distintas etapas de creación. Es evidente que no nos encontramos con el laborioso trabajo de intentar reconstruir un arquetipo a partir de copias corruptas, sino que es un proceso lineal en el que la edición representa el texto más depurado.

A este respecto, todas las variae lectiones que podemos examinar son perfectamente ilustrativas del proceso de creación literaria, donde es normal que el autor (en este caso, el traductor) revise su escrito y lo vaya modificando con el fin de corregir imperfecciones y lograr un texto más elegante. Es una tarea laboriosa, aconsejada, como es sabido, entre otros, por Horacio (ars. 289294), autor a quien conoció bien y tradujo Graciliano Afonso ${ }^{32}$.

En este caso la labor de corrección, tal y como refería el propio traductor y atendiendo a los testimonios manuscritos conservados, comenzó con la redacción de una obra en verso $(E)$ a partir de un texto en prosa; este texto se lima y se pasa luego a limpio $(M)$, sea por el propio autor ${ }^{33}$ o por otra persona

32. Cf. Alberto Blecua, op. cit., pp. 117-122. Un lúcido artículo sobre el proceso de redacción de las obras en la Antigüedad y el Renacimiento, con abundantes referencias, es el de José M. ${ }^{a}$ Maestre Maestre, "Limae labor y creación literaria en latín durante el Renacimiento: las dos versiones del Carmen in natali Serenissimi Philippi de Sobrarias», pp. 135 en José Ma Maestre Maestre - Joaquín Pascual Barea (coord.), op. cit., t. I, pp. 135-178.

33. Es curioso observar que Agustín Millares Carlo - Manuel Hernández Suárez (op. cit., p. 41) apuntan a que sólo el libro $4 .^{\circ}$ que se encuentra en El Museo Canario tiene correcciones del traductor. 
(en nuestro caso, la edad del traductor hace fácil suponer la existencia de uno o varios amanuenses que realizaran la tarea, demostrado esto, como se dijo, por el color diferente de la tinta). Evidentemente, todas las correcciones llevadas a cabo son una unidad que al analizarlas permiten diferenciar varios estratos (los que distinguimos con $E_{1}$ y $E_{2}$ ). Este tipo de correcciones que aparecen en los borradores conservados debe diferenciarse de las realizadas posteriormente por el autor, estadio que corresponde a las erratas y enmiendas que se hallan al final del segundo tomo de la edición impresa.

El problema que surge en la tradición textual de esta obra es la existencia en la edición de 1854 de lecturas diferentes a las que ofrecen los borradores $E$ y $M$. Ello obliga a plantear por lo menos dos hipótesis: a) la existencia de otro manuscrito que fuera una copia en limpio del texto de la traducción -lógico es considerar que fuera revisado por el traductor- $y$, por tanto, el texto definitivo sobre el que se hiciera la edición; o b) que el traductor corrigiera sobre las galeradas de dicha edición, lo cual debió hacerse en esta fase del proceso editorial. Sin embargo, parece más razonable pensar que se diera aquí el primero de los supuestos, dado el elevado coste que supondría para nuestro traductor la corrección de pruebas de imprenta en un texto que de por sí le había ocasionado muchos sinsabores económicos ${ }^{34}$. No obstante, gracias a todo esto, muchas personas, sobre todo de las Islas, tuvieron la suerte de leer en "verso endecasílabo» una de las más grandes obras de la Antigüedad Clásica.

34. Diferentes cartas entre el impresor Collina y don Graciliano revelan las dificultades monetarias que soportó nuestro autor en su empeńo de publicar esta traducción, que, por lo que se puede suponer, pagó de su peculio. Cf. Agustín Millares Carlo - Manuel Hernández Suárez, op. cit., p. 72. 